\title{
Effects of dietary strawberry powder on blood lipids and inflammatory markers in obese human subjects
}

\author{
Susan J. Zunino ${ }^{1 *}$, Mardi A. Parelman ${ }^{2}$, Tammy L. Freytag ${ }^{1}$, Charles B. Stephensen ${ }^{1}$, Darshan S. Kelley ${ }^{1}$, \\ Bruce E. Mackey ${ }^{3}$, Leslie R. Woodhouse ${ }^{1}$ and Ellen L. Bonnel ${ }^{2}$ \\ ${ }^{1}$ United States Department of Agriculture, Agricultural Research Service, Western Human Nutrition Research Center, \\ 430 West Health Sciences Drive, Davis, CA 95616, USA \\ ${ }^{2}$ Department of Nutrition, University of California, Davis, One Shields Avenue, Davis, CA 95616, USA \\ ${ }^{3}$ United States Department of Agriculture, Agricultural Research Service, Pacific West Area, 800 Buchanan Street, Albany, \\ CA 94710, USA
}

(Submitted 3 June 2011 - Final revision received 17 August 2011 - Accepted 8 October 2011 - First published online 9 November 2011)

\begin{abstract}
Obesity is a strong risk factor for the development of CVD, hypertension and type 2 diabetes. The overall goal of the present pilot study was to feed strawberries, in the form of freeze-dried powder, to obese subjects to determine whether dietary strawberries beneficially altered lipid profiles and reduced blood markers of inflammation compared with a control intervention. A total of twenty healthy subjects (thirteen females and seven males) aged between 20 and 50 years with a BMI between 30 and $40 \mathrm{~kg} / \mathrm{m}^{2}$ completed the present 7 -week double-blind, randomised, cross-over trial. Each subject received a prepared diet $7 \mathrm{~d} /$ week for 7 weeks consisting of approximately $35 \%$ of energy from fat, $20 \%$ protein, $45 \%$ carbohydrate and $14 \mathrm{~g}$ fibre. Blood was collected on days 1 and 8 for baseline information. After the first week, subjects were randomly assigned to the strawberry powder (equivalent to four servings of frozen strawberries) or control (strawberry-flavoured) intervention for 3 weeks. For the remaining 3 weeks, subjects crossed over to the opposite intervention. Blood was collected again at the end of weeks 3, 4, 6 and 7. A comprehensive chemistry panel, lipid profile analyses and measurement of inflammatory mediators were performed for each blood draw. A 3-week dietary intervention with strawberry powder reduced plasma concentrations of cholesterol and small HDL-cholesterol particles, and increased LDL particle size in obese subjects $(P<0 \cdot 05)$. Dietary strawberry powder reduced risk factors for CVD, stroke and diabetes in obese volunteers, suggesting a potential role for strawberries as a dietary means to decrease obesity-related disease.
\end{abstract}

Key words: Strawberries: Obesity: Lipids: Inflammation

Obesity constitutes a serious public health problem in the USA as well as in other parts of the world ${ }^{(1,2)}$. Obesity rates have steadily risen over the past 20 years, and $>30 \%$ of US adults are obese according to a recent analysis of the 2007-8 National Health and Nutrition Examination Survey data ${ }^{(1)}$. Health care expenses associated with obesity have reached nearly 147 billion dollars ${ }^{(3)}$. Obesity is a strong risk factor for the development of a number of chronic diseases which include hypertension, CVD and stroke, and the metabolic syndrome leading to insulin resistance and type 2 diabetes mellitus $^{(4)}$. Hypercholesterolaemia and dyslipidaemia are prominent in obese individuals. The development of type 2 diabetes in obese individuals further increases the risk of CVD due to metabolic disturbances resulting from insulin resistance and increased levels of inflammatory molecules. Atherosclerotic plaque formation can be initiated by an injury to the endothelial cell lining in the arterial wall by hypertension and subendothelial accumulation of oxidised, or otherwise modified, $\mathrm{LDL}^{(5)}$. As a consequence of this damage, immune cells such as monocytes and T-lymphocytes enter the subendothelium and macrophages engulf the modified LDL creating foam cells, which eventually build up to form the plaques. The inflammation mediated by activated immune cells leads to the continued migration of macrophages and T-lymphocytes into the plaques, remodelling of the arterial wall and eventual blockage of the blood vessel.

Adipose tissue is a source of the inflammatory cytokines IL- 6, TNF- $\alpha$ and IL-1 $\beta$ which stimulate the production of

Abbreviations: CRP, C-reactive protein; ICAM, intercellular adhesion molecule; ICP-AES, inductively coupled plasma atomic emission spectrometry; ORAC, oxygen radical absorbance capacity; TAS, total antioxidant status; UCDMC, University of California Davis Medical Center; VCAM, vascular adhesion molecule.

*Corresponding author: Dr S. J. Zunino, fax +1 530752 5271, email susan.zunino@ars.usda.gov 
acute-phase proteins, such as C-reactive protein (CRP), fibrinogen and serum amyloid $\mathrm{A}^{(6-11)}$. Increased levels of serum CRP and serum amyloid A have been linked to an increased expression of endothelial adhesion molecules and chemoattractants that promote recruitment of immune cells into the

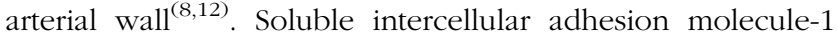
(ICAM-1) and soluble vascular adhesion molecule-1 (VCAM-1) both increase in the blood of obese humans, and are associated with an increased risk of $\mathrm{CVD}^{(13,14)}$. IL-8, one of the chemoattractant molecules involved in atherosclerosis, can be produced by adipocytes, and circulating concentrations of IL-8 increase in obesity ${ }^{(15)}$. Obesity leads to a prothrombotic state from the increased production of fibrinogen and other coagulation factors ${ }^{(9)}$. Adipose tissue produces the hormone leptin, the obese (Ob) gene product, and elevated plasma levels of leptin are strongly associated with insulin-resistant states found in obesity, type 2 diabetes and hypertension ${ }^{(16)}$. Increased leptin production in obese individuals can enhance the inflammatory processes associated with the development of atherosclerotic plaques and has been linked to the development of other chronic inflammatory diseases ${ }^{(16,17)}$.

Epidemiological and clinical studies have shown a positive association between dietary berries and reduced CVD risk (reviewed in Basu et $a l^{(18)}$ ). Strawberries are a fibre- and Zn-rich food that contain abundant amounts of vitamin $\mathrm{C}$ and antioxidant polyphenols ${ }^{(19,20)}$. Fibre is well known to reduce serum cholesterol and the risk of $\mathrm{CVD}^{(21)}$. Plasma $\mathrm{Zn}$ is inversely correlated with inflammation and atherosclerosis, and obese individuals generally present with decreased plasma $\mathrm{Zn}$ concentrations ${ }^{(22-24)}$. Strawberries contain high levels of flavonoid antioxidants including catechins, anthocyanins, and the flavanols quercetin and kaempferol, all of which have anti-inflammatory abilities ${ }^{(19)}$. In the present pilot study, the overall goal was to determine whether dietary strawberries fed in the form of freeze-dried powder to obese subjects would reduce risk factors for CVD and other health problems known to be associated with morbidity and mortality of obese individuals. The specific hypothesis was that dietary strawberries would reduce cholesterol due to the increased intake of fibre, and beneficially alter lipid profiles and reduce blood markers of inflammation.

\section{Methods}

\section{Subject recruitment}

The study was conducted according to the guidelines laid down in the Declaration of Helsinki and all procedures involving human subjects were approved by the University of California Davis, Institutional Review Board. Written informed consent was obtained from all subjects. Healthy male and female subjects who participated in the present study were 20-50 years old with a BMI of $30-40 \mathrm{~kg} / \mathrm{m}^{2}$. Subjects were recruited by advertisements, flyers and press releases, and screened by telephone questionnaire for health history, lifestyle, physical activity levels and dietary habits. For screening purposes, blood pressure, complete blood count and differential leucocyte counts were measured at the Western Human
Nutrition Research Center (WHNRC). The pathology laboratory at the University of California Davis Medical Center (UCDMC) measured blood lipids and performed chemistry panels. All samples sent to the UCDMC pathology laboratory were coded and de-identified. Inclusion criteria for the present study were blood pressure, blood cell counts and blood chemistry profiles within the normal range, and commitment to the dietary intervention and scheduled testing. Exclusion criteria were vegetarianism, current use of tobacco products, drinking more than one alcoholic beverage per d $(29.6 \mathrm{ml}$ distilled liquor, $88.7 \mathrm{ml}$ wine and $354.9 \mathrm{ml}$ beer), use of cholesterollowering medications, steroids for asthma or other conditions, thyroid-regulating drugs or weight-loss products. Subjects with mildly or moderately elevated total cholesterol $(<6.5$ mmol/l) or TAG $(<3.4 \mathrm{mmol} / \mathrm{l})$ were not excluded. Subjects taking oral contraceptives were not excluded.

\section{Study design and dietary intervention}

The study was a 7-week double-blind, randomised, cross-over trial. Each subject received a prepared diet of three meals per $\mathrm{d}, 7 \mathrm{~d} /$ week for the duration of the study. Menu rotation was every $4 \mathrm{~d}$. Energy intake was prescribed per individual based on the Mifflin-St Jeor equation ${ }^{(25)}$ corrected for an appropriate activity factor. The background diet consisted of approximately 35\% energy from fat (animal and plant), 20\% protein, $45 \%$ carbohydrate and $14 \mathrm{~g}$ fibre, which typifies the average American diet (National Health and Nutrition Examination Survey III) ${ }^{(26,27)}$ and was low in fruits and vegetables (less than four servings per $\mathrm{d}$ ). The subjects came to the WHNRC for breakfast and dinner Monday to Friday (except for holidays), and received a packaged lunch for those days. For weekends and holidays, all meals were packaged and given to the subjects to consume. The subjects were asked to return all uneaten foods. Intervention with strawberries, in the form of strawberry powder, occurred at breakfast and dinner and was supervised, except for weekends and holidays. The California Strawberry Commission (Watsonville, CA, USA) provided the strawberry powder that was produced from individually quick frozen kosher, conventional (nonorganic) whole strawberries. The strawberries were supplied by Anacapa Foods (Watsonville, CA, USA) and Frozsun Foods (Watsonville, CA, USA). The powder was prepared and packaged by Van Drunen Farms (Momence, IL, USA) by a commercial food freeze-drying process with a moisture content of approximately $5 \%$. The strawberry powder was maintained at $-20^{\circ} \mathrm{C}$ during the study. The mixture of strawberries used to generate the powder contained the University of California public cultivars Camarosa (37\%), Ventana (13\%) and Diamante (13\%), and two proprietary varieties (37\%) in production in 2004. In the present study, one serving of frozen strawberries ( $80 \mathrm{~g}$ ) was used to calculate the amount of powder needed per serving. The subjects consumed the equivalent of four servings of strawberries per $d$. The strawberry intervention consisted of two servings of strawberry powder mixed as a milkshake, in yogurt, cream cheese, or water-based, sweetened beverage. The control intervention was the same drinks or food products with strawberry 
flavouring and red food colour (McCormick \& Company, Hunt Valley, MD, USA). The flavourings were imitation strawberry extract (McCormick) and Kool-Aid strawberry artificial flavour (Kraft Foods, Rye Brook, NY, USA). Additional energy from the strawberries was matched in the control drink by adding an equivalent amount of glucose, fructose and sucrose. In the first week of the study, subjects received prepared meals without intervention. Blood was collected on day 1 and at the end of the first week to obtain baseline information. After the first week, subjects were randomised into one of two groups. Group 1 received the strawberry intervention and group 2 received the control intervention for 3 weeks. For the remaining 3 weeks of the study, subjects crossed over to the opposite intervention. All subjects fasted $12 \mathrm{~h}$ before each blood draw. Blood was collected again at the end of weeks 3, 4, 6 and 7 during the study. Measurements for each blood draw included a complete blood count with the differential leucocyte count and a comprehensive chemistry panel. Body weights were measured daily and dietary energy adjusted when necessary to maintain the same body weight throughout the study. Blood pressure, heart rate and body temperature were measured at the time of screening and at each blood draw during the study.

\section{Chemistry and lipid panels}

For serum preparation, blood was collected in Vacutainer tubes (Becton Dickinson, Franklin Lakes, NJ, USA), allowed to clot for $30 \mathrm{~min}$ at room temperature, and centrifuged at $4^{\circ} \mathrm{C}, 10 \mathrm{~min}, 1300 \mathrm{~g}$. Serum samples were sent by courier to the UCDMC pathology laboratory the day of the blood draw. Alanine aminotransferase was added to the panel by the pathology laboratory midway through the study and analysed for ten subjects.

\section{Plasma zinc measurements}

Blood was collected into syringes containing $\mathrm{Zn}$-free lithium heparin (S-Monovette for trace metal analysis; Sarstedt, Numbrecht, Germany), centrifuged at $1300 \mathrm{~g}, 4^{\circ} \mathrm{C}, 10 \mathrm{~min}$, and stored at $-80^{\circ} \mathrm{C}$ before analysis. Plasma $\mathrm{Zn}$ concentrations were determined by inductively coupled plasma atomic emission spectrometry (ICP-AES) using a Vista AX CCD simultaneous ICP-AES analyser with an SPS5 autosampler (Varian, Inc., Walnut Creek, CA, USA). Zn standards and plasma samples were prepared in $1 \mathrm{M}-\mathrm{HNO}_{3}$ (Fisher Scientific, Pittsburg, PA, USA), centrifuged at $2200 \mathrm{~g}, 4^{\circ} \mathrm{C}, 15 \mathrm{~min}$, and the supernatants recovered for ICP-AES analysis. Qualitycontrol samples included a serum reference with known $\mathrm{Zn}$ value (Seronorm Trace Elements Serum LI; Accurate Chemical and Scientific Corporation, Westbury, NY, USA), and an internal quality-control plasma (Utak Laboratories, Inc., Valencia, CA, USA). Bovine liver standard, a certified reference material (no. SRM 1577b; National Institute of Standards and Technology, Boulder, CO, USA), was run as an ICP-AES Zn reference control. All blanks, standards and samples were run in duplicate.

\section{Lipid particle analysis}

Lipid size and particle analyses were performed on plasma samples using NMR at Liposcience, Inc. (Raleigh, NC, USA) ${ }^{(28)}$. Plasma was prepared using EDTA-Vacutainer tubes (Becton Dickinson) centrifuged at $4^{\circ} \mathrm{C}, 10 \mathrm{~min}, 1300 \mathrm{~g}$. All plasma samples sent to Liposcience were frozen at $-80^{\circ} \mathrm{C}$ and shipped on dry ice.

\section{Acute-phase proteins and inflammatory markers}

Serum and plasma were prepared as described above and frozen at $-80^{\circ} \mathrm{C}$ until analysis of inflammatory markers. CRP was measured in serum using a high sensitivity test kit and Immulite instrumentation (Siemens Healthcare Diagnostics, Inc., Deerfield, IL, USA). Complement 3c was measured in serum on the Cobas Integra 400 Plus chemical analyser (Roche Diagnostics, Indianapolis, IN, USA) using kit reagents from Roche Diagnostics. Enzyme-linked immunosorbant assays were used to measure serum amyloid A (US Biological, Swampscott, MA, USA) and plasma fibrinogen (AssayPro, St Charles, MO, USA) following the manufacturers' protocols. Plasma levels of IL-1 $\beta$, IL-6, IL-8, TNF- $\alpha$, leptin, soluble ICAM- 1 and soluble VCAM-1 were measured using Milliplex detection kits (Millipore Corporation, St Charles, MO, USA) run on a Bioplex multiplex instrument (BioRad, Hercules, CA, USA). Samples were run in duplicate.

\section{Antioxidant status}

Serum was prepared as described above and measured for antioxidant status using the total antioxidant status (TAS) and oxygen radical absorbance capacity (ORAC) assays. TAS was measured on the Cobas Integra 400 Plus chemical analyser using reagents from Randox Laboratories (San Diego, CA, USA) and following the manufacturer's recommendations. ORAC values were measured using the method described by Prior et al. ${ }^{(29)}$. The radical generator 2,2'-azobis(2-methylproprionamidine)dihydrochloride (AAPH) and 6-hydroxy-2,5,7,8-tetramethylchroman-2carboxylic acid (Trolox) were purchased from Sigma (St Louis, MO, USA). Fluorescein sodium salt was obtained from Acros Organics (Morris Plains, NJ, USA). The loss of the fluorescein signal due to oxidation was monitored for $2 \mathrm{~h}$ at $37^{\circ} \mathrm{C}$ on a SpectraMAX GeminiXS spectrofluorometer running SoftMAX PRO version 4.0 software (Molecular Devices, Sunnyvale, CA, USA). Trolox equivalents were calculated by comparing the areas under the curves (fluorescence intensity $v$. time) of Trolox standards to serum samples.

Butylated hydroxytoluene was added as a preservative to plasma used for 8-isoprostane analyses. Concentrations of free 8-isoprostane were measured by enzyme-linked immunoassay kits (Cayman Chemical Company, Ann Arbor, MI, USA) following the manufacturer's recommendations. Assays for TAS and 8-isoprostane were run in duplicate. ORAC assays were run in triplicate. 


\section{Statistical analyses}

The Statistical Analysis Systems statistical software package version 9.2 (SAS Institute, Cary, NC, USA) was used for statistical analyses using the Proc Mixed procedure to fit the cross-over model with the baseline values (blood draws 1 and 2) as the covariates. The means of the two baselines were used as covariates to account for a greater amount of variability among the subjects, and thus, reduced error and increased precision in the tests. The Means procedure was used for means and standard deviations. Data subsets were transformed using Box-Cox power transformations, if needed to conform to a normal distribution. When a carry-over effect was detected, an analysis was performed that included only the first 3-week intervention period of the study. We expected cholesterol to be reduced by dietary strawberry powder due to the addition of fibre to the diet from the powder ${ }^{(20)}$. Therefore, a one-tailed test was used only for the analysis of total cholesterol. All data are presented as means and standard deviations to indicate variability of individual values, with significance at $P<0.05$.

\section{Results}

\section{Recruitment and study participation}

The recruitment, screening and participation of subjects are shown in Fig. 1. The age, weight and BMI for female and male subjects who completed the screening process and the study are presented in Table 1. Of the thirty-one subjects who participated, eleven dropped out for the following reasons: dietary (dislike of provided meals, five subjects); medical (unrelated to the study, two subjects); personal (undisclosed, two subjects); schedule conflicts (two subjects). Energy was adjusted in the diets weekly if needed to maintain body weight throughout the study period. Compliance was

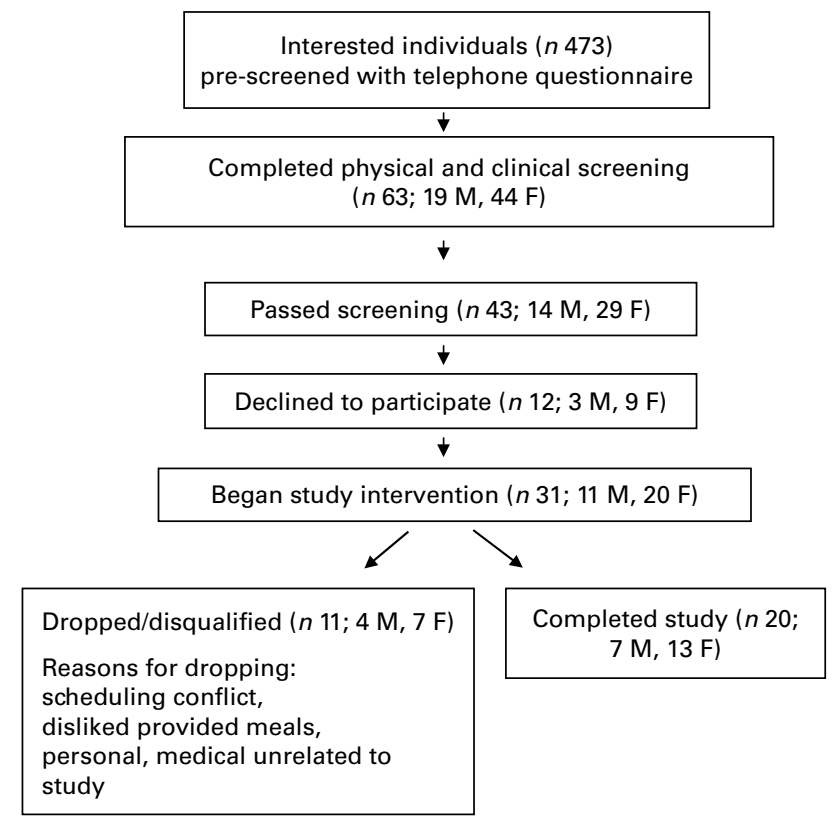

Fig. 1. Recruitment, screening and participation of the study subjects. $\mathrm{M}$, male; F, female.
Table 1. Age, weight and BMI of subjects completing screening and the study

(Mean values and standard deviations)

\begin{tabular}{|c|c|c|c|c|}
\hline & \multicolumn{2}{|c|}{$\begin{array}{l}\text { Completed } \\
\text { screening }\end{array}$} & \multicolumn{2}{|c|}{$\begin{array}{l}\text { Completed } \\
\text { study }\end{array}$} \\
\hline & Mean & SD & Mean & SD \\
\hline \multicolumn{5}{|l|}{ Female } \\
\hline & \multicolumn{2}{|c|}{44} & \multicolumn{2}{|c|}{13} \\
\hline Age (years) & $31 \cdot 1$ & $10 \cdot 8$ & $31 \cdot 8$ & 11.4 \\
\hline Weight (kg) & $96 \cdot 0$ & $13 \cdot 3$ & $96 \cdot 1$ & $9 \cdot 1$ \\
\hline BMI $\left(\mathrm{kg} / \mathrm{m}^{2}\right)$ & $35 \cdot 6$ & $4 \cdot 3$ & $35 \cdot 6$ & 3.0 \\
\hline \multicolumn{5}{|l|}{ Male } \\
\hline$n$ & \multicolumn{2}{|c|}{19} & \multicolumn{2}{|c|}{7} \\
\hline Age (years) & $29 \cdot 9$ & 8.5 & 29.4 & $6 \cdot 6$ \\
\hline Weight (kg) & 111.8 & $16 \cdot 4$ & 104.6 & $11 \cdot 3$ \\
\hline BMI $\left(\mathrm{kg} / \mathrm{m}^{2}\right)$ & 34.4 & 3.4 & $32 \cdot 3$ & $2 \cdot 1$ \\
\hline
\end{tabular}

assured at least $5 \mathrm{~d} /$ week of the study, since the subjects were fed the interventions at breakfast and dinner under supervision at the Center on weekdays.

\section{Vitals, blood chemistry and lipid panels}

No differences were observed between the intervention groups for blood pressure, heart rates or body temperature. Blood chemistry and lipid parameters were measured for each blood draw by the pathology laboratory at the UCDMC (Table 2). Diet effects were observed for $\mathrm{Na}, \mathrm{CO}_{2}$ and total cholesterol. Dietary strawberry powder reduced $\mathrm{Na}$ and $\mathrm{CO}_{2}$ concentrations in the blood compared with the control group $(P<0 \cdot 05)$. There was a carry-over (order) effect for serum cholesterol into the second intervention period of the cross-over design, and intervention period 1 only was analysed for this parameter (ten subjects). Serum cholesterol was reduced in the subjects receiving the strawberry powder compared with the control group $(P=0 \cdot 0438)$. There was no effect of blood draw (week $2 v$. week 3) or a diet $\times$ blood draw interaction for any parameter shown in Table 2 .

\section{Lipid particle profiles by NMR analysis}

Profiles of lipid particles were obtained for each blood draw (Table 3). There was evidence of a carry-over (order) effect for total HDL, small HDL particles, VLDL size, LDL size, HDL size, intermediate-density lipoprotein-cholesterol and small HDLcholesterol, and intervention period 1 only was analysed for these parameters (ten subjects). All other parameters had an $n$ 20. There was a diet effect for small HDL particle concentrations, LDL size and small HDL-cholesterol concentrations. Dietary strawberry powder reduced the concentrations of small HDL particles and small HDL-cholesterol, and increased the mean particle size of LDL $(P<0 \cdot 05)$. There was no effect of blood draw (week $2 v$. week 3 ) or a diet $\times$ blood draw interaction.

\section{Inflammatory markers and antioxidant status}

Plasma or serum was analysed for inflammatory markers and antioxidant status (Table 4). The acute-phase protein 
Table 2. Chemistry and lipid panels

(Unadjusted mean values and standard deviations (n 20 except for alanine aminotransferase (ALT) $\dagger$ and total cholesterol $\ddagger)$ )

\begin{tabular}{|c|c|c|c|c|c|c|c|c|c|c|c|c|c|}
\hline \multirow[b]{2}{*}{ Parameter } & \multicolumn{2}{|c|}{ Baseline 1} & \multicolumn{2}{|c|}{ Baseline 2} & \multicolumn{2}{|c|}{ Control wk $2 \S$} & \multicolumn{2}{|c|}{ Strawberry wk 2} & \multicolumn{2}{|c|}{ Control wk 3} & \multicolumn{2}{|c|}{ Strawberry wk 3} & \multirow[b]{2}{*}{$P \|$} \\
\hline & Mean & SD & Mean & SD & Mean & SD & Mean & SD & Mean & SD & Mean & SD & \\
\hline Alkaline phosphatase $(\mathrm{U} / \mathrm{l})$ & $62 \cdot 05$ & $16 \cdot 41$ & $61 \cdot 70$ & $15 \cdot 32$ & $62 \cdot 21$ & 14.77 & $61 \cdot 30$ & $15 \cdot 39$ & $63 \cdot 20$ & $16 \cdot 19$ & $61 \cdot 30$ & $16 \cdot 63$ & $0 \cdot 15$ \\
\hline AST (U/l) & 23.00 & 5.46 & 22.65 & $10 \cdot 77$ & $20 \cdot 42$ & $5 \cdot 17$ & 20.50 & $6 \cdot 24$ & $20 \cdot 80$ & $7 \cdot 23$ & 21.65 & $7 \cdot 27$ & 0.97 \\
\hline $\operatorname{ALT}(U / 1) \dagger$ & $24 \cdot 20$ & $10 \cdot 11$ & $23 \cdot 60$ & 13.50 & $21 \cdot 70$ & 11.08 & 21.80 & $10 \cdot 41$ & $22 \cdot 30$ & $12 \cdot 27$ & $24 \cdot 20$ & $16 \cdot 25$ & 0.08 \\
\hline Creatinine $(\mu \mathrm{mol} / \mathrm{l})$ & 74.12 & 10.04 & $74 \cdot 21$ & $12 \cdot 28$ & 71.56 & 9.19 & $72 \cdot 22$ & $10 \cdot 46$ & 74.57 & 11.84 & 74.26 & 12.63 & 0.84 \\
\hline Total bilirubin $(\mu \mathrm{mol} / \mathrm{l})$ & 11.88 & 3.74 & 13.77 & 3.62 & 11.52 & $2 \cdot 74$ & 11.46 & 3.42 & 11.63 & 3.42 & 12.48 & 3.64 & 0.40 \\
\hline $\mathrm{Na}(\mathrm{mmol} / \mathrm{l})$ & 139.40 & 1.82 & 138.00 & 2.03 & $138 \cdot 84^{a}$ & 2.03 & $137 \cdot 90^{\mathrm{b}}$ & 1.74 & $138.40^{\mathrm{a}}$ & 1.82 & $137 \cdot 50^{\mathrm{b}}$ & $2 \cdot 12$ & 0.02 \\
\hline $\mathrm{K}(\mathrm{mmol} / \mathrm{l})$ & 4.38 & 0.61 & 4.48 & 1.03 & 4.26 & 0.51 & 4.21 & 0.28 & $4 \cdot 17$ & 0.30 & 4.39 & 0.55 & 0.26 \\
\hline Chloride $(\mathrm{mmol} / \mathrm{l})$ & 103.60 & $2 \cdot 58$ & 103.40 & 2.62 & $103 \cdot 63$ & 2.41 & $103 \cdot 50$ & 3.12 & $103 \cdot 20$ & 2.33 & 103.00 & $2 \cdot 13$ & 0.68 \\
\hline $\mathrm{CO}_{2}(\mathrm{mmol} / \mathrm{l})$ & 26.55 & 1.99 & 26.65 & 3.08 & $27 \cdot 21^{a}$ & 2.08 & $26 \cdot 00^{\mathrm{b}}$ & $2 \cdot 13$ & $27 \cdot 00^{\mathrm{a}}$ & $2 \cdot 41$ & $26 \cdot 60^{\mathrm{b}}$ & $2 \cdot 28$ & 0.02 \\
\hline Urea N (mmol/l) & 4.62 & 0.92 & 4.52 & 1.02 & 4.57 & 0.97 & 4.70 & 1.07 & 4.50 & 0.99 & 4.34 & 0.84 & 0.97 \\
\hline Glucose $(\mathrm{mmol} / \mathrm{l})$ & 4.82 & 0.69 & 4.57 & 0.72 & 4.73 & 0.67 & 4.65 & 0.53 & $4 \cdot 76$ & 0.59 & 4.65 & 0.53 & 0.22 \\
\hline $\mathrm{Ca}(\mathrm{mmol} / \mathrm{l})$ & $2 \cdot 29$ & 0.08 & $2 \cdot 33$ & 0.06 & $2 \cdot 31$ & 0.07 & $2 \cdot 28$ & 0.06 & $2 \cdot 30$ & 0.07 & $2 \cdot 29$ & 0.09 & $0 \cdot 17$ \\
\hline Protein $(\mathrm{g} / \mathrm{l})$ & 67.75 & 4.42 & 67.80 & 3.83 & 67.58 & 3.98 & 65.85 & 4.48 & $67 \cdot 10$ & 3.45 & 66.80 & 3.82 & 0.09 \\
\hline Albumin $(\mathrm{g} / \mathrm{l})$ & 39.60 & 3.09 & 40.00 & 3.04 & 39.74 & 2.51 & 38.95 & 3.20 & 39.55 & 3.22 & 39.55 & 3.03 & 0.26 \\
\hline Total cholesterol $(\mathrm{mmol} / \mathrm{l}) \ddagger$ & 4.72 & 0.98 & 4.72 & 1.06 & $4 \cdot 63^{\mathrm{a}}$ & 1.14 & $4.42^{b}$ & 1.05 & $4 \cdot 51^{\mathrm{a}}$ & 0.96 & $4 \cdot 37^{b}$ & 0.91 & 0.04 \\
\hline Total HDL-cholesterol ( $\mathrm{mmol} / \mathrm{l})$ & 1.09 & 0.29 & $1 \cdot 10$ & 0.31 & 1.01 & 0.24 & 1.01 & 0.24 & 1.03 & 0.20 & 1.05 & 0.26 & 0.68 \\
\hline Total LDL-cholesterol (mmol/l) & 3.14 & 0.84 & 3.11 & 0.83 & 2.97 & 0.86 & 2.97 & 0.90 & 2.92 & 0.82 & 2.95 & 0.82 & 0.31 \\
\hline TAG $(\mathrm{mmol} / \mathrm{l})$ & 1.07 & 0.43 & 1.13 & 0.65 & 1.17 & 0.57 & 1.22 & 0.66 & $1 \cdot 12$ & 0.51 & 1.13 & 0.55 & 0.97 \\
\hline
\end{tabular}

wk, Week; AST, aspartat

${ }^{a, b}$ Mean values with unlike superscript letters in overall diet effect between the two intervention groups were significantly different $(P<0.05)$.

"Baseline data (blood draws 1 and 2) were used as covariates.

TALT was added to the chemistry panel by the UC Davis Medical Center during the second half of the study and analysis was performed for ten study subjects.

¥A carry-over (order) effect was observed for cholesterol and the data presented represent a one-tailed, period 1 analysis $(n 10)$

\$Wk 2 data represent 2 weeks or intervention (blood draws 3 and 5), and wk 3 data represent 3 weeks of intervention (blood draws 4 and 6 ) with the control diet (control) or the diet containing the strawberry powder (strawberry).

$\| P$ value for the overall diet effect between the two intervention groups. No diet $\times$ blood draw interactions were found. 
Table 3. Lipid particle concentrations and size*

(Unadjusted mean values and standard deviations ( $n 20$ except as noted in footnote $\dagger$ ))

\begin{tabular}{|c|c|c|c|c|c|c|c|c|c|c|c|c|c|}
\hline \multirow[b]{2}{*}{ Parameter } & \multicolumn{2}{|c|}{ Baseline 1} & \multicolumn{2}{|c|}{ Baseline 2} & \multicolumn{2}{|c|}{ Control wk $2 \ddagger$} & \multicolumn{2}{|c|}{ Strawberry wk 2} & \multicolumn{2}{|c|}{ Control wk 3} & \multicolumn{2}{|c|}{ Strawberry wk 3} & \multirow[b]{2}{*}{$P \S$} \\
\hline & Mean & SD & Mean & SD & Mean & SD & Mean & SD & Mean & SD & Mean & SD & \\
\hline \multicolumn{14}{|l|}{ Particle concentrations } \\
\hline Total VLDL and chylomicrons (nmol/l) & $52 \cdot 85$ & $23 \cdot 22$ & $54 \cdot 70$ & 21.45 & $56 \cdot 65$ & $20 \cdot 70$ & $57 \cdot 72$ & $22 \cdot 65$ & $56 \cdot 33$ & $22 \cdot 21$ & 53.98 & $21 \cdot 26$ & 0.78 \\
\hline Large VLDL and chylomicrons (nmol//) & 1.78 & 1.75 & 2.34 & 3.42 & $2 \cdot 86$ & 2.99 & 2.74 & 3.91 & $2 \cdot 25$ & 2.74 & 2.57 & 2.97 & 0.80 \\
\hline Medium VLDL (nmol/l) & $17 \cdot 82$ & $10 \cdot 99$ & $18 \cdot 91$ & 9.87 & 22.54 & $14 \cdot 13$ & $22 \cdot 61$ & $10 \cdot 72$ & $22 \cdot 11$ & $15 \cdot 59$ & $22 \cdot 65$ & $15 \cdot 20$ & 0.87 \\
\hline Small VLDL (nmol/l) & $33 \cdot 26$ & $14 \cdot 20$ & 33.45 & 14.38 & 31.25 & $12 \cdot 85$ & 32.37 & 12.99 & 31.97 & 11.87 & $28 \cdot 77$ & 11.39 & 0.53 \\
\hline Total LDL (nmol/l) & $1110 \cdot 92$ & 366.97 & $1065 \cdot 51$ & $416 \cdot 86$ & $1069 \cdot 68$ & 384.62 & 1097.96 & $467 \cdot 12$ & $1086 \cdot 62$ & $345 \cdot 16$ & $1079 \cdot 32$ & $442 \cdot 64$ & 0.48 \\
\hline IDL (nmol/I) & $42 \cdot 28$ & 55.92 & 37.61 & 48.02 & 30.48 & 34.71 & $36 \cdot 28$ & 43.68 & 32.39 & $35 \cdot 62$ & $34 \cdot 21$ & 50.95 & 0.68 \\
\hline Large LDL (nmol//) & 394.90 & $220 \cdot 81$ & 375.55 & $205 \cdot 34$ & 311.99 & $188 \cdot 21$ & 342.79 & $189 \cdot 50$ & 330.64 & 175.92 & 329.08 & $202 \cdot 84$ & 0.79 \\
\hline Small LDL (nmol/l) & $673 \cdot 75$ & $304 \cdot 27$ & $652 \cdot 35$ & 393.25 & $727 \cdot 21$ & $347 \cdot 25$ & $718 \cdot 88$ & 418.05 & 723.59 & $327 \cdot 11$ & 716.03 & 407.93 & 0.40 \\
\hline Medium small LDL (nmol/l) & $146 \cdot 66$ & $55 \cdot 40$ & $137 \cdot 87$ & 74.55 & $153 \cdot 67$ & 62.07 & 149.82 & $68 \cdot 14$ & $156 \cdot 54$ & 63.39 & $149 \cdot 81$ & 85.53 & 0.29 \\
\hline Very small LDL (nmol/l) & 527.09 & $250 \cdot 56$ & 514.48 & 321.80 & 573.54 & 289.25 & 569.07 & 348.52 & 567.05 & 266.09 & $566 \cdot 22$ & 326.03 & 0.41 \\
\hline Total HDL $(\mu \mathrm{mol} / \mathrm{l}) \dagger$ & 29.25 & 3.62 & $28 \cdot 16$ & 3.29 & $28 \cdot 80$ & 2.08 & 26.96 & 3.69 & $28 \cdot 77$ & 2.75 & 28.95 & $4 \cdot 18$ & 0.13 \\
\hline Large HDL $(\mu \mathrm{mol} / /)$ & $5 \cdot 70$ & 2.99 & $6 \cdot 13$ & 3.48 & 5.56 & 3.09 & $5 \cdot 72$ & 2.86 & $5 \cdot 39$ & 2.59 & 6.02 & $2 \cdot 71$ & 0.14 \\
\hline Medium HDL ( $(\mu \mathrm{mol} / \mathrm{l})$ & $5 \cdot 18$ & $2 \cdot 82$ & 4.01 & 3.27 & 3.44 & 2.41 & 3.68 & $2 \cdot 62$ & 3.27 & 2.49 & 4.04 & 3.39 & 0.65 \\
\hline Small HDL $(\mu \mathrm{mol} / \mathrm{l}) \dagger$ & $18 \cdot 30$ & 4.38 & 18.02 & 3.99 & $19 \cdot 67^{\mathrm{a}}$ & 3.63 & $17 \cdot 21^{\mathrm{b}}$ & 3.84 & $20 \cdot 04^{a}$ & $5 \cdot 19$ & $18 \cdot 14^{\mathrm{b}}$ & 5.20 & 0.043 \\
\hline \multicolumn{14}{|l|}{ Mean particle sizes } \\
\hline VLDL size $(\mathrm{nm}) \dagger$ & $48 \cdot 23$ & $6 \cdot 18$ & $48 \cdot 01$ & $5 \cdot 83$ & $51 \cdot 39$ & $5 \cdot 18$ & 47.55 & 4.54 & $50 \cdot 14$ & $7 \cdot 11$ & $47 \cdot 80$ & $5 \cdot 27$ & 0.20 \\
\hline LDL size $(\mathrm{nm}) \dagger$ & 21.05 & 0.70 & 21.02 & 0.70 & $20 \cdot 60^{\mathrm{b}}$ & 0.62 & $21 \cdot 22^{\mathrm{a}}$ & 0.64 & $20 \cdot 70^{\mathrm{b}}$ & 0.55 & $21 \cdot 13^{\mathrm{a}}$ & 0.63 & 0.043 \\
\hline HDL size $(n m) \dagger$ & 8.88 & 0.44 & 8.92 & 0.47 & 8.73 & 0.37 & 9.02 & 0.50 & $8 \cdot 70$ & 0.37 & 9.03 & 0.42 & 0.08 \\
\hline \multicolumn{14}{|l|}{ Mass concentrations } \\
\hline Total VLDL and chylomicron TAG (mg/l) & $588 \cdot 6$ & $314 \cdot 0$ & $635 \cdot 6$ & $407 \cdot 3$ & $730 \cdot 0$ & $431 \cdot 6$ & $724 \cdot 2$ & 451.5 & $676 \cdot 9$ & $406 \cdot 3$ & $697 \cdot 6$ & $416 \cdot 9$ & 0.88 \\
\hline Large VLDL and chylomicron TAG (mg/l) & $192 \cdot 8$ & $151 \cdot 0$ & $228 \cdot 1$ & $270 \cdot 7$ & 265.5 & $228 \cdot 2$ & 265.5 & $308 \cdot 2$ & $222 \cdot 8$ & $226 \cdot 6$ & 249.5 & $240 \cdot 2$ & 0.92 \\
\hline Medium VLDL TAG (mg/l) & $246 \cdot 3$ & $161 \cdot 8$ & $257 \cdot 6$ & $152 \cdot 4$ & 324.5 & $222 \cdot 0$ & $313 \cdot 2$ & $167 \cdot 0$ & $309 \cdot 3$ & $244 \cdot 3$ & 318.9 & $245 \cdot 1$ & 0.98 \\
\hline Small VLDL TAG $(\mathrm{mg} / \mathrm{l})$ & $149 \cdot 4$ & $62 \cdot 0$ & $149 \cdot 8$ & $62 \cdot 7$ & $140 \cdot 0$ & $56 \cdot 0$ & $145 \cdot 5$ & $56 \cdot 0$ & $144 \cdot 8$ & 51.9 & $129 \cdot 2$ & 50.5 & 0.55 \\
\hline Total LDL-cholesterol (mg/l) & $1000 \cdot 6$ & $330 \cdot 7$ & 949.9 & $323 \cdot 1$ & $901 \cdot 8$ & 308.4 & $945 \cdot 9$ & $350 \cdot 8$ & 933.3 & $270 \cdot 3$ & $922 \cdot 2$ & $340 \cdot 0$ & 0.71 \\
\hline IDL-cholesterol $(\mathrm{mg} / \mathrm{l}) \dagger$ & 52.4 & $69 \cdot 3$ & $46 \cdot 6$ & $59 \cdot 5$ & 53.9 & $53 \cdot 0$ & $20 \cdot 5$ & $20 \cdot 3$ & $52 \cdot 1$ & $50 \cdot 1$ & $20 \cdot 5$ & $30 \cdot 3$ & 0.09 \\
\hline Large LDL-cholesterol (mg/l) & 533.2 & $299 \cdot 1$ & 503.9 & $273 \cdot 8$ & $419 \cdot 4$ & $255 \cdot 0$ & 461.6 & $257 \cdot 9$ & $447 \cdot 6$ & $237 \cdot 3$ & 443.5 & 274.0 & 0.82 \\
\hline Small LDL-cholesterol (mg/l) & $415 \cdot 1$ & 176.9 & $399 \cdot 3$ & $232 \cdot 3$ & $444 \cdot 6$ & 199.9 & $439 \cdot 3$ & 235.1 & 445.5 & $192 \cdot 9$ & $436 \cdot 3$ & $245 \cdot 6$ & 0.32 \\
\hline Medium small LDL-cholesterol (mg/l) & $159 \cdot 2$ & $59 \cdot 8$ & $149 \cdot 3$ & 80.5 & $166 \cdot 6$ & $67 \cdot 0$ & $162 \cdot 3$ & $73 \cdot 1$ & $169 \cdot 9$ & $68 \cdot 8$ & $162 \cdot 4$ & $93 \cdot 2$ & 0.29 \\
\hline Very small LDL-cholesterol $(\mathrm{mg} / \mathrm{l})$ & 255.9 & $118 \cdot 2$ & $250 \cdot 1$ & $153 \cdot 7$ & $278 \cdot 0$ & $135 \cdot 8$ & $277 \cdot 1$ & $163 \cdot 0$ & $275 \cdot 6$ & $125 \cdot 7$ & 273.9 & 154.9 & 0.47 \\
\hline Total HDL-cholesterol $(\mathrm{mg} / \mathrm{l})$ & 434.4 & $103 \cdot 4$ & 428.4 & $106 \cdot 1$ & $408 \cdot 0$ & 71.9 & $411 \cdot 1$ & $88 \cdot 2$ & $400 \cdot 3$ & $66 \cdot 3$ & $430 \cdot 6$ & $82 \cdot 1$ & 0.16 \\
\hline Large HDL-cholesterol (mg/l) & $186 \cdot 2$ & $108 \cdot 7$ & $197 \cdot 8$ & $125 \cdot 2$ & $176 \cdot 4$ & 104.5 & 182.5 & $103 \cdot 6$ & $169 \cdot 1$ & $91 \cdot 1$ & $190 \cdot 6$ & $96 \cdot 4$ & 0.22 \\
\hline Medium HDL-cholesterol (mg/l) & $67 \cdot 0$ & 36.5 & 51.9 & $42 \cdot 3$ & 44.5 & $31 \cdot 1$ & $47 \cdot 6$ & $33 \cdot 8$ & $42 \cdot 4$ & $32 \cdot 2$ & $52 \cdot 3$ & 43.9 & 0.65 \\
\hline Small HDL-cholesterol $(\mathrm{mg} / \mathrm{l}) \dagger$ & $181 \cdot 1$ & $43 \cdot 2$ & $178 \cdot 7$ & $39 \cdot 2$ & $194 \cdot 6^{\mathrm{a}}$ & $36 \cdot 4$ & $170 \cdot 5^{\mathrm{b}}$ & 37.9 & $198 \cdot 3^{\mathrm{a}}$ & 51.6 & $180 \cdot 1^{\mathrm{b}}$ & $51 \cdot 2$ & 0.046 \\
\hline
\end{tabular}

wk, Week; IDL, intermediate-density lipoprotein

a,b Mean values with unlike superscript letters in overall diet effect between the two intervention groups were significantly different $(P<0.05)$.

*Baseline data (blood draws 1 and 2) were used as covariates.

†A carry-over (order) effect was observed for total HDL, small HDL, VLDL size, LDL size, HDL size, IDL-cholesterol and small HDL-cholesterol, and the data presented for these parameters are from intervention period 1 ( $n$ 10). A A carameters, $n 20$.

intervention (blood draws 3 and 5), and wk 3 data represent 3 weeks of intervention (blood draws 4 and 6) with the control diet (control) or the diet containing the strawberry powder (strawberry).

$\S P$ value for the overall diet effect between the two intervention groups. No diet $\times$ blood draw interactions were found. 
Table 4. Inflammatory markers and antioxidant status in serum and plasma*

(Unadjusted mean values and standard deviations ( $n 20$ except as noted in footnote $\dagger$ ))

\begin{tabular}{|c|c|c|c|c|c|c|c|c|c|c|c|c|c|}
\hline \multirow[b]{2}{*}{ Parameters } & \multicolumn{2}{|c|}{ Baseline 1} & \multicolumn{2}{|c|}{ Baseline 2} & \multicolumn{2}{|c|}{ Control wk $2 \ddagger$} & \multicolumn{2}{|c|}{ Strawberry wk 2} & \multicolumn{2}{|c|}{ Control wk 3} & \multicolumn{2}{|c|}{ Strawberry wk 3} & \multirow[b]{2}{*}{$P \S$} \\
\hline & Mean & SD & Mean & SD & Mean & SD & Mean & SD & Mean & SD & Mean & SD & \\
\hline \multicolumn{14}{|l|}{ Inflammatory markers } \\
\hline $\mathrm{IL}-1 \beta(\mathrm{ng} / \mathrm{l})$ & 0.80 & 1.45 & 0.55 & 1.07 & 0.84 & 1.68 & 0.81 & 1.47 & 0.72 & 1.41 & 0.81 & 1.60 & 0.99 \\
\hline IL-6 (ng/l) & $6 \cdot 14$ & 8.26 & 5.63 & 7.62 & 6.38 & $7 \cdot 67$ & 6.09 & 7.45 & 5.54 & 6.34 & 5.88 & 6.81 & 0.57 \\
\hline IL-8 (ng/l) & 2.04 & 1.36 & 1.74 & 1.29 & $2 \cdot 10$ & 1.70 & 1.95 & 1.60 & 1.87 & 1.43 & $2 \cdot 14$ & 1.77 & 0.73 \\
\hline TNF- $\alpha$ (ng/l) & $3 \cdot 28$ & 1.86 & 2.99 & 1.45 & 3.44 & 1.87 & 3.13 & 1.50 & $3 \cdot 30$ & 2.06 & $3 \cdot 32$ & $2 \cdot 13$ & 0.45 \\
\hline Complement 3c $(\mu \mathrm{g} / \mathrm{l})$ & 154.96 & 22.94 & $162 \cdot 87$ & 21.72 & $160 \cdot 15$ & $27 \cdot 20$ & 161.03 & $22 \cdot 87$ & 154.69 & $17 \cdot 71$ & 162.56 & $30 \cdot 23$ & 0.24 \\
\hline sICAM-1 $(\mu \mathrm{g} / \mathrm{l}) \dagger$ & $163 \cdot 72$ & $61 \cdot 28$ & 164.91 & 68.46 & $176 \cdot 13$ & 109.54 & 152.85 & $25 \cdot 67$ & 183.37 & 99.23 & $164 \cdot 67$ & $22 \cdot 38$ & 0.26 \\
\hline sVCAM-1 $(\mu \mathrm{g} / \mathrm{l}) \dagger$ & 1018.55 & $256 \cdot 18$ & $973 \cdot 29$ & 278.99 & 939.94 & 301.08 & $991 \cdot 10$ & 321.32 & $1004 \cdot 35$ & 259.98 & $1024 \cdot 27$ & $226 \cdot 56$ & 0.19 \\
\hline Leptin $(\mu \mathrm{g} / \mathrm{l})$ & 37.97 & 23.56 & $35 \cdot 15$ & 23.83 & 34.04 & $22 \cdot 62$ & 34.60 & 23.89 & 33.72 & $25 \cdot 12$ & 33.25 & 22.45 & 0.85 \\
\hline CRP $(\mathrm{mg} / \mathrm{l})$ & 3.48 & 2.63 & 3.89 & 3.04 & 3.08 & 2.62 & 3.81 & 3.51 & 3.49 & 2.90 & 3.59 & 2.31 & 0.36 \\
\hline $\mathrm{SAA}(\mathrm{mg} / \mathrm{l})$ & $5 \cdot 41$ & 4.54 & 5.84 & $4 \cdot 16$ & 5.99 & 4.61 & 5.35 & 3.74 & $6 \cdot 27$ & 6.00 & 6.97 & 4.84 & 0.70 \\
\hline Fibrinogen (g/l) & 3.23 & 0.95 & 3.38 & $1 \cdot 10$ & $3.08^{\mathrm{b}}$ & 1.03 & $3 \cdot 16^{\mathrm{a}}$ & 1.14 & $2 \cdot 97^{\mathrm{b}}$ & 0.86 & $3 \cdot 30^{\mathrm{a}}$ & 1.17 & 0.049 \\
\hline $\mathrm{Zn}(\mu \mathrm{mol} / \mathrm{l})$ & 10.99 & $1 \cdot 16$ & 11.90 & 1.41 & 11.69 & 0.99 & $11 \cdot 27$ & 1.33 & $11 \cdot 88$ & 1.38 & 11.53 & 1.31 & 0.78 \\
\hline \multicolumn{14}{|l|}{ Antioxidant status } \\
\hline ORAC $(\mu \mathrm{mol} / \mathrm{l} \mathrm{TE}) \dagger$ & $873 \cdot 21$ & 164.45 & 903.49 & 92.07 & 920.75 & $58 \cdot 20$ & $870 \cdot 30$ & $92 \cdot 84$ & $906 \cdot 07$ & $50 \cdot 35$ & $857 \cdot 14$ & $114 \cdot 28$ & 0.26 \\
\hline TAS $(\mathrm{mmol} / \mathrm{l})$ & 1.72 & 0.21 & $1 \cdot 70$ & 0.25 & 1.68 & 0.21 & 1.70 & 0.22 & 1.69 & 0.23 & $1 \cdot 71$ & 0.19 & 0.27 \\
\hline 8-Isoprostane (ng/l) & $62 \cdot 67$ & $15 \cdot 43$ & 63.02 & 14.04 & 61.55 & 12.97 & $62 \cdot 18$ & $15 \cdot 46$ & $60 \cdot 11$ & 14.02 & $62 \cdot 00$ & $16 \cdot 87$ & 0.49 \\
\hline
\end{tabular}

wk, Week; sICAM-1, soluble intercellular adhesion molecule 1; sVCAM-1, soluble vascular adhesion molecule 1; CRP, C-reactive protein; SAA, serum amyloid A; ORAC, oxygen radical absorbance capacity; TE, Trolox equivalents; TAS, total antioxidant status.

a,b Mean values with unlike superscript letters in overall diet effect between the two intervention groups were significantly different $(P<0.05)$.

* Baseline data (blood draws 1 and 2) were used as covariates.

†A carry-over (order) effect was observed for sICAM-1, sVCAM-1 and ORAC and the data presented represent a period 1 analysis $(n 10)$. For all other parameters, $n 20$.

$\ddagger$ Wk 2 data represent 2 weeks of intervention (blood draws 3 and 5), and wk 3 data represent 3 weeks of intervention (blood draws 4 and 6 ) with the control diet (control) or the diet containing the strawberry powder (strawberry).

$\S P$ value for the overall diet effect between the two intervention groups. No diet $\times$ blood draw interactions were found. 
fibrinogen was slightly increased in subjects consuming the strawberry powder compared with the control diet $(P<0.05)$, but the concentrations remained within the normal range. There were no differences observed for the other inflammatory markers. There were no differences in antioxidant status between the two dietary groups as measured by the ORAC, TAS or 8-isoprostane assay. There was no effect of blood draw or a diet $\times$ blood draw interaction for these measurements.

\section{Discussion}

The goal of the present pilot study was to determine the potential of dietary strawberries in the form of freeze-dried powder to improve markers of health in obese human subjects who are at high risk for developing serious diseases, such as CVD, stroke and diabetes. Hypercholesterolaemia is common in obese individuals. In the present study, we did not specifically recruit individuals with elevated cholesterol, and the mean total cholesterol concentrations for our participants was 4.66 (SD 0.89$) \mathrm{mmol} / \mathrm{l}$ at the time of screening. However, consumption of the strawberry powder (equivalent to four servings of frozen strawberries per d) reduced plasma concentrations of total cholesterol by about $4 \%$ compared with the control diet. This reduction was observed by 2 weeks of feeding and was maintained through week 3. The relatively small decrease in total cholesterol in response to strawberry powder in the present study may be because of the normal cholesterol concentrations before the supplementation, and the short duration of the intervention periods. In general, it should be noted that the subjects in the present study were relatively healthy obese individuals. In recent studies, Basu et al ${ }^{(30,31)}$ fed strawberry powder for 4 or 8 weeks to subjects who had both the metabolic syndrome and elevated plasma cholesterol. The powder was mixed as a beverage and equivalent to about four servings of strawberries per $d$. After 4 weeks of the strawberry powder intervention, these authors observed a $5 \%$ decrease in total plasma cholesterol $^{(30)}$. In a separate study, a $10 \%$ reduction in total cholesterol was observed after an 8-week intervention with the same serving size of strawberry powder ${ }^{(31)}$. Fibre is well known to reduce cholesterol ${ }^{(21)}$. Reduction in total cholesterol may have been at least partly due to the increased intake of fibre in the diet from the addition of the strawberry powder. Strawberries have approximately $2 \mathrm{~g}$ fibre/100 $\mathrm{g}$ fresh edible portion $^{(19,20)}$. In the present study, each subject received about $8 \mathrm{~g}$ of extra fibre per $\mathrm{d}$ during the 3 weeks on the strawberry-enriched diet. Taken together, these data suggest that prolonged consumption of strawberries may continue to improve the cholesterol-lowering effect of this fruit over time.

Basu et al. ${ }^{(30,31)}$ noted a 6 and $11 \%$ reduction in the concentration of LDL-cholesterol after 4- and 8-week interventions, respectively. Jenkins et $a l^{(32)}$ also reported a reduction in LDL-cholesterol and in the ratio of total:HDL-cholesterol after feeding $454 \mathrm{~g}$ strawberries per $\mathrm{d}$ for 1 month. In the present study, concentrations of LDL-cholesterol were not different between the dietary groups. However, our analyses of lipid particles by NMR revealed a reduction in the concentration of small HDL particles and small HDL-cholesterol in subjects consuming the strawberry powder. These lipid fractions have been linked to health status. Evaluation of HDL particle sizes in patients with coronary artery disease revealed a significantly higher concentration of small HDL particles in the coronary artery disease group than in control subjects $^{(33)}$. Also, higher concentrations of small HDL-cholesterol have been observed in Asian Indian men who have a greater incidence of CVD compared with Caucasian men ${ }^{(34)}$, suggesting a link between CVD risk and the presence of small HDL. Patients with acute ischaemic stroke had greater concentrations of small HDL particles, as well as small LDL particles, than healthy controls ${ }^{(35)}$. Furthermore, both LDL and HDL particle distribution and size have been linked to the metabolic syndrome and insulin resistance. As part of the Genetics of Coronary Artery Disease in Alaska Natives study, analyses of lipid distribution in Alaskan Eskimos with the metabolic syndrome showed an increase in the concentration of intermediate-density lipoprotein and small HDL particles compared with subjects without the metabolic syndrome ${ }^{(36)}$. In 2005, Festa et al. ${ }^{(37)}$ reported the NMR-generated lipid profiles of prediabetic subjects as part of the Insulin Resistance Atherosclerosis Study. An increase in the concentration of small HDL was observed in subjects who had become diabetic at follow-up compared with non-diabetic patients at the time of follow-up.

Although the present study showed no change in LDL concentrations between the dietary groups, the strawberry intervention increased the mean particle size of plasma LDL. Decreased LDL size is positively associated with an increased risk of developing CVD, the metabolic syndrome and type 2 diabetes ${ }^{(37-40)}$. Festa et al. $^{(37)}$ showed that a reduction of $0.3-0.5 \mathrm{~nm}$ in the diameter of LDL was positively correlated with the development of type 2 diabetes. Smaller HDL and larger VLDL particle sizes have also been associated with an increased risk of $\mathrm{CVD}^{(41,42)}$. There was a potential trend towards an increase in HDL particle size in the present study, and this difference may have attained significance if a carry-over effect had not been observed. The present data indicate that a 3-week intervention with strawberry powder was able to reduce plasma concentrations of small HDL particles and cholesterol and increase LDL particle size in healthy obese subjects, and, thereby, reduce risk factors for obesity-related disease.

There were no differences in inflammatory markers, plasma $\mathrm{Zn}$ or antioxidant status of the serum from our volunteers between the two dietary groups. Basu et al. ${ }^{(31)}$ observed a decrease in circulating VCAM-1 in subjects with the metabolic syndrome receiving freeze-dried strawberry powder for 8 weeks. In an epidemiological study over a 10.9-year followup period involving the Women's Health Study, women who consumed two servings or greater of strawberries per week $v$. none had a $14 \%$ lower likelihood of an elevated CRP equal to or above $3 \mathrm{mg} / \mathrm{l}^{(43)}$. A 16-week study with progressive doses of cranberry juice resulted in decreased plasma concentrations of ICAM-1-1 and VCAM- ${ }^{(44)}$. However, Erlund et al. ${ }^{(45)}$ observed no changes in soluble ICAM-1 levels in the blood after feeding a combination of berry preparations (including 
strawberries) for 8 weeks. Strawberries have recently been shown to decrease plasma levels of CRP and IL-6 in response to a high-fat meal, indicating that dietary strawberries have the potential for modulating immediate inflammatory responses induced by dietary fat ${ }^{(46)}$. In the present study, 3 weeks of dietary intervention with strawberries may not have been long enough to observe differences in inflammatory markers between the two dietary groups. Antioxidant status was shown to increase in several clinical trials with consumption of strawberries or other berries, in either postprandial or longer-term protocols (reviewed in Basu et al. ${ }^{(18)}$ ). Recent studies using oxidised LDL as a marker have shown that dietary strawberries increased serum antioxidant capacity both postprandially ${ }^{(47)}$ and after 3 and 4 weeks of strawberry intake $^{(32,48)}$. Using three different tests for antioxidant potential or oxidation status, no difference in serum antioxidant status was observed in the present study. However, the subjects fasted for approximately $12 \mathrm{~h}$ before each blood draw, and antioxidant potential may not have been maintained at a steady-state level in our volunteers under these conditions. The strengths of the present study included the control of dietary intake by feeding all foods to the participants and maintaining body weight throughout the study. Therefore, the variables of dietary and weight change were reduced with this protocol. The use of freeze-dried strawberry powder rather than fresh strawberries further reduced seasonal variations in the fruit during the study. Analysis of drying methods has shown comparable levels of phenolics, vitamin $C$ and antioxidant activity between fresh and freeze-dried strawberries ${ }^{(49)}$. Furthermore, the cross-over design allowed the subjects to be their own controls, and the dietary effects of the strawberry powder were observed within a 3-week period. Some parameters that were measured showed a carry-over or order effect, so that only the first intervention period was used to evaluate significant effects. The study design would have been enhanced with a washout period between the cross-over intervention periods as well as longer intervention periods. Increasing the number of participants may also have enhanced the study outcomes. However, the alterations in lipid subfractions that were observed may represent a beneficial reduction in risk factors for CVD, stroke and the metabolic syndrome/diabetes in our obese volunteers, suggesting a role for strawberries as a dietary means to decrease obesity-related disease. The present study provides data to support larger and more demographically diverse clinical trials with strawberries and other fruits to define the roles they may play in reducing morbidity and mortality associated with obesity.

\section{Acknowledgements}

This study was supported by the United States Department of Agriculture (USDA) Current Research Information System project no. 5306-51530-013-00D and 5306-51 530-018-00D, and a grant from the California Strawberry Commission. S. J. Z. is a member of the scientific advisory committee for the California Strawberry Commission. The authors report no conflict of interest. The authors' contributions were as follows:
S. J. Z., C. B. S., D. S. K. and E. L. B. designed the research; T. L. F. and L. R. W. conducted the research; S. J. Z., M. A. P. and B. E. M. analysed the data; S. J. Z. wrote the manuscript with input from all authors and had primary responsibility for the final content. All authors read and approved the final manuscript. USDA is an equal opportunity provider and employer.

\section{References}

1. Flegal KM, Carroll MD, Ogden CL, et al. (2010) Prevalence and trends in obesity among US adults, 1999-2008. JAMA 303, 235-241.

2. Nguyen DM \& El-Serag HB (2010) The epidemiology of obesity. Gastroenterol Clin North Am 39, 1-7.

3. Finkelstein EA, Trogdon JG, Cohen JW, et al. (2009) Annual medical spending attributable to obesity: payer- and servicespecific estimates. Health Aff (Millwood) 28, w822-w831.

4. Samartin S \& Chandra RK (2001) Obesity, overnutrition and the immune system. Nutr Res 21, 243-262.

5. Libby P (2002) Inflammation in atherosclerosis. Nature $\mathbf{4 2 0}$, $868-872$.

6. Hotamisligil GS, Arner P, Caro JF, et al. (1995) Increased adipose tissue expression of tumor necrosis factor-alpha in human obesity and insulin resistance. J Clin Invest $\mathbf{9 5}$, 2409-2415.

7. Mohamed-Ali V, Goodrick S, Rawesh A, et al. (1997) Human subcutaneous adipose tissue releases IL6 but not TNF-alpha in vivo. J Clin Endocrinol Metab 82, 4196-4200.

8. O'Brien KD \& Chait A (2006) Serum amyloid A; the "other" inflammatory protein. Curr Atheroscler Rep 8, 62-68.

9. Faber DR, de Groot PG \& Visseren FL (2009) Role of adipose tissue in haemostasis, coagulation and fibrinolysis. Obesity Res 10, 554-563.

10. Rosenson RS \& Koenig W (2003) Utility of inflammatory markers in the management of coronary artery disease. Am J Cardiol 92, 10-18.

11. Fain JN (2006) Release of interleukins and other inflammatory cytokines by human adipose tissue is enhanced in obesity and primarily due to the nonfat cells. Vitam Horm 74, 443-477.

12. Pickup JC (2004) Inflammation and activated innate immunity in the pathogenesis of type 2 diabetes. Diabetes Care 27, 813-823.

13. Straczkowski M, Lewczuk $\mathrm{P}$, Dzienis-Straczkowski S, et al. (2002) Elevated soluble intercellular adhesion molecule-1 levels in obesity: relationship to insulin resistance and tumor necrosis factor-alpha system activity. Metabolism $\mathbf{5 1}$, $75-78$.

14. Shai I, Pischon T, Hu FB, et al. (2006) Soluble intercellular adhesion molecules, soluble vascular cell adhesion molecules, and risk of coronary heart disease. Obesity 14, 2099-2106.

15. Kim C-S, Park H-S, Kawada T, et al. (2006) Circulating levels of MCP-1 and IL- 8 are elevated in human obese subjects and associated with obesity-related parameters. Int $J$ Obes 30, 1347-1355.

16. Otero M, Lago R, Lago F, et al. (2005) Leptin, from fat to inflammation: old question and new insights. FEBS Lett 579, 295-301.

17. Lago R, Gómez R, Lago F, et al. (2008) Leptin beyond body weight regulation - current concepts concerning its role in immune function and inflammation. Cell Immunol 252, $139-145$. 
18. Basu A, Rhone M \& Lyons TJ (2010) Berries: emerging impact on cardiovascular health. Nutr Rev 68, 168-177.

19. Hannum SM (2004) Potential impact of strawberries on human health: a review of the science. Crit Rev Food Sci Nutr 44, 1-17.

20. United States Department of Agriculture, Agricultural Research Service (2009) National nutrient database for standard reference, release 22. http://www.ars.usda.gov/main/ site_main.htm?modecode $=12-35-45-00$ (accessed 24 September 2010)

21. Erkkilä AT \& Lichtenstein AH (2006) Fiber and cardiovascular disease risk: how strong is the evidence? J Cardiovasc Nurs 21, 3-8.

22. Bao B, Prasad AS, Beck FWJ, et al. (2010) Zinc decreases C-reactive protein, lipid peroxidation, and inflammatory cytokines in elderly subjects: a potential implication of zinc as an atheroprotective agent. Am J Clin Nutr 91, 1634-1641.

23. Costarelli L, Muti E, Malavolta M, et al. (2010) Distinctive modulation of inflammatory and metabolic parameters in relation to zinc nutritional status in adult overweight/obese subjects. J Nutr Biochem 21, 432-437.

24. Yang YJ, Choi BY, Chun BY, et al. (2010) Dietary zinc intake is inversely related to subclinical atherosclerosis measured by carotid intima-media thickness. BrJ Nutr 104, 1202-1211.

25. Mifflin MD, St Jeor ST, Hill LA, et al. (1990) A new predictive equation for resting energy expenditure in healthy individuals. Am J Clin Nutr 51, 241-247.

26. Centers for Disease Control and Prevention (1994) Daily dietary fat and total food-energy intakes - Third National Health and Nutrition Examination Survey, Phase 1, 198891. MMWR Morb Mortal Wkly Rep 43, 116-117, 123-125.

27. United States Department of Health and Human Services, National Center for Health Statistics (1996) The Third National Health and Nutrition Examination Survey (NHANES III, 1988-94) Reference Manuals and Reports. NCHS CD-ROM. Hyattsville, MD: Centers for Disease Control and Prevention.

28. Jeyarajah EJ, Cromwell WC \& Otvos JD (2006) Lipoprotein particle analysis by nuclear magnetic resonance spectroscopy. Clin Lab Med 26, 847-870.

29. Prior RL, Hoang H, Gu L, et al. (2003) Assays for hydrophilic and lipophilic antioxidant capacity (oxygen radical absorbance capacity (ORAC(FL)) of plasma and other biological and food samples. J Agric Food Chem 51, 3273-3279.

30. Basu A, Wilkinson M, Penugonda K, et al. (2009) Freezedried strawberry powder improves lipid profile and lipid peroxidation in women with metabolic syndrome: baseline and post intervention effects. Nutr J 8, 43-49.

31. Basu A, Fu DX, Wilkinson M, et al. (2010) Strawberries decrease atherosclerotic markers in subjects with metabolic syndrome. Nutr Res 30, 462-469.

32. Jenkins DJA, Nguyen TH, Kendall CWC, et al. (2008) The effect of strawberries in a cholesterol-lowering dietary portfolio. Metabolism 57, 1636-1644.

33. Cheung MC, Brown BG, Wolf AC, et al. (1991) Altered particle size distribution of apolipoprotein A-I-containing lipoproteins in subjects with coronary artery disease. J Lipid Res 32, 383-394.

34. Bhalodkar NC, Blum S, Rana T, et al. (2004) Comparison of levels of large and small high-density lipoprotein cholesterol in Asian Indian men compared with Caucasian men in the Framington Offspring Study. Am J Cardiol 94, 1561-1563.

35. Zeljkovic A, Vekic J, Spasojevic-Kalimanovska V, et al. (2010) LDL and HDL subclasses in acute ischemic stroke: prediction of risk and short-term mortality. Atherosclerosis $\mathbf{2 1 0}$, 548-554.

36. Rivellese AA, Patti L, Kaufman D, et al. (2008) Lipoprotein particle distribution and size, insulin resistance, and metabolic syndrome in Alaska Eskimos: The GOCADAN Study. Atherosclerosis 200, 350-358.

37. Festa A, Williams K, Hanley AJG, et al. (2005) Nuclear magnetic resonance lipoprotein abnormalities in prediabetic subjects in the Insulin Resistance Atherosclerosis Study. Circulation 111, 3465-3472.

38. Koba S, Yokota Y, Hirano T, et al. (2008) Small LDL-cholesterol is superior to LDL-cholesterol for determining severe coronary atherosclerosis. $J$ Atherosclerosis Thromb $\mathbf{1 5}$ $250-260$.

39. Garvey WT, Kwon S, Zheng D, et al. (2003) Effects of insulin resistance and type 2 diabetes on lipoprotein subclass particle size and concentration determined by nuclear magnetic resonance. Diabetes 52, 453-462.

40. Rizzo M, Pernice V, Frasheri A, et al. (2009) Small, dense low-density lipoproteins (LDL) are predictors of cardioand cerebro-vascular events in subjects with the metabolic syndrome. Clin Endocrinol 70, 870-875.

41. Lyons TJ, Jenkins AJ, Zheng D, et al. (2006) Nuclear magnetic resonance-determined lipoprotein subclass profile in the DCCT/EDIC cohort: associations with carotid intimamedia thickness. Diabet Med 23, 955-966.

42. Arsenault BJ, Lemieux I, Despres JP, et al. (2009) HDL particle size and the risk of coronary heart disease in apparently healthy men and women: the EPIC-Norfolk prospective population study. Atherosclerosis 206, 276-281.

43. Sesso HD, Gaziano JM, Jenkins DJ, et al. (2007) Strawberry intake, lipids, C-reactive protein, and the risk of cardiovascular disease in women. J Am Coll Nutr 26, 303-310.

44. Ruel G, Pomerleau S, Couture P, et al. (2008) Low-calorie cranberry juice supplementation reduces plasma oxidized LDL and cell adhesion molecule concentrations in men. Br J Nutr 99, 352-359.

45. Erlund I, Koli R, Alfthan G, et al. (2008) Favorable effects of berry consumption on platelet function, blood pressure, and HDL cholesterol. Am J Clin Nutr 87, 323-331.

46. Edirisinghe I, Banaszewski K, Cappozzo J, et al. (2011) Strawberry anthocyanin and its association with postprandial inflammation and insulin. Br J Nutr 106, 913-922.

47. Burton-Freeman B, Linares A, Hyson D, et al. (2010) Strawberry modulates LDL oxidation and postprandial lipemia in response to high-fat meal in overweight hyperlipidemic men and women. J Am Coll Nutr 29, 46-54.

48. Henning SM, Seeram NP, Zhang Y, et al. (2010) Strawberry consumption is associated with increased antioxidant capacity in serum. J Med Food 13, 116-122.

49. Wojdyło A, Figiel A \& Oszmiański J (2009) Effect of drying methods with the application of vacuum microwaves on the bioactive compounds, color, and antioxidant activity of strawberry fruits. J Agric Food Chem 57, 1337-1343. 